\title{
Impact of PI3K/AKT/mTOR pathway activation on the prognosis of patients with head and neck squamous cell carcinomas
}

\author{
Darío García-Carracedo ${ }^{1, *}$, Maria Ángeles Villaronga ${ }^{2, *}$, Saúl Álvarez-Teijeiro², \\ Francisco Hermida-Prado², Iñigo Santamaría ${ }^{3}$, Eva Allonca², Laura Suárez- \\ Fernández ${ }^{2}$, Maria Victoria Gonzalez ${ }^{4}$ Milagros Balbín ${ }^{3}$, Aurora Astudillo ${ }^{4}$, Pablo \\ Martínez-Camblor ${ }^{5}$, Gloria H. Su${ }^{1,6,7}$, Juan Pablo Rodrigo², Juana María García- \\ Pedrero $^{2}$

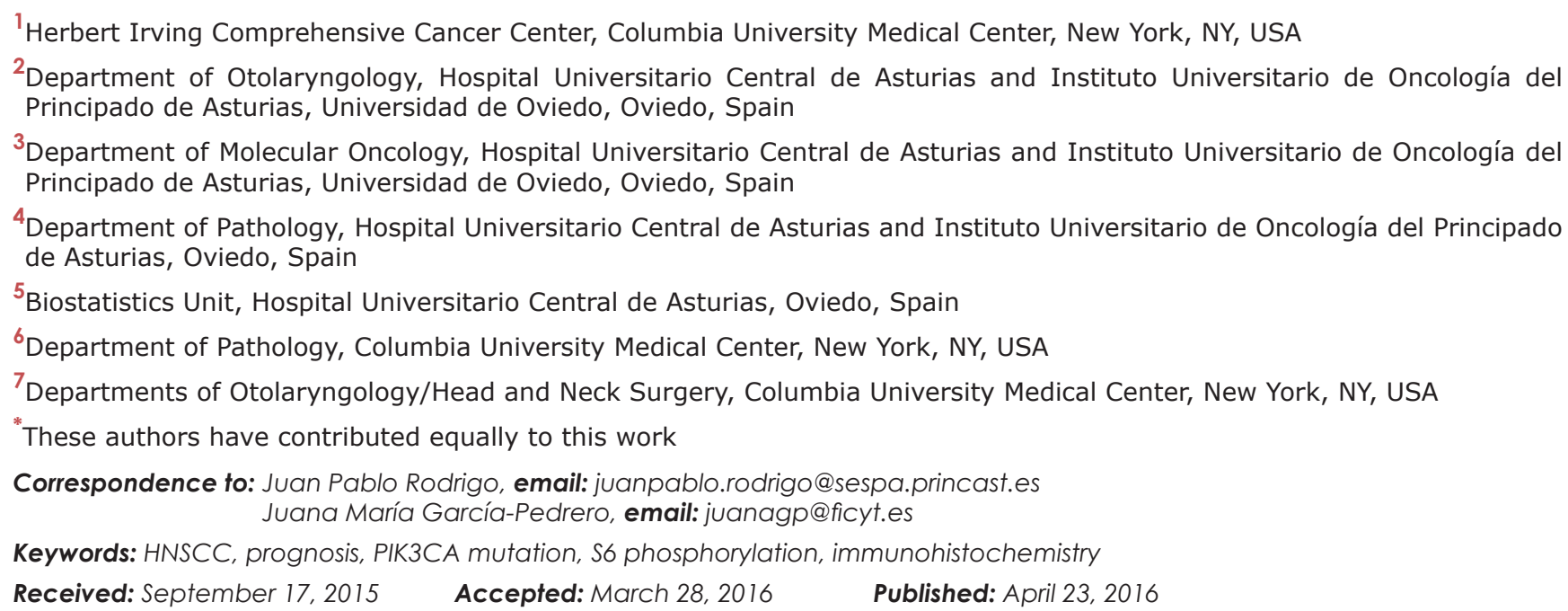

\section{ABSTRACT}

The PI3K/AKT/mTOR signaling pathway has emerged as one of the most frequently deregulated in head and neck squamous cell carcinomas (HNSCC). Numerous alterations of various upstream and downstream components have been described; however, their prognostic significance and impact on HNSCC patient survival remains to be established. This was addressed using an unbiased cohort of 93 consecutive and homogeneous surgically treated HNSCC patients and results confirmed in 432 HNSCC patients. Our findings reveal the high prevalence of $\mathbf{S 6}$ phosphorylation, a surrogate marker of mTORC1 activation, in HNSCC specimens (>70\%) and, more importantly, demonstrate its relevance on clinical outcome. Phosphorylation of ribosomal protein S6 on either Ser235/236 or Ser240/244 was consistently and significantly correlated with favorable prognosis, although with differences depending on the tumor site. Thus, $\mathbf{p}-\mathbf{S 6}$ expression was significantly correlated with better disease-specific survival specifically in the subgroup of laryngeal carcinoma patients $(P<0.001)$. In addition, multivariate regression models revealed $\mathrm{p}-\mathrm{S} 6$ to be an inverse and independent predictor of lymph-node metastasis $(P=0.004)$ and distant metastasis $(P=0.006)$. Taken together, this study unveils an unprecedented correlation of $m$ TOR activation with improved clinical outcome in patients with laryngeal carcinomas and uncovers the potential of p-S6 expression as a good prognostic biomarker and an inverse predictor of lymph node and distant metastases. These results should be of broad interest as immunohistochemical detection of p-S6 may help to stratify patients and guide treatment decisions. 


\section{INTRODUCTION}

Head and neck squamous cell carcinoma (HNSCC) is the sixth most common cancer worldwide. The term HNSCC is applied primarily to those cancers arising from the squamous epithelium of the oral cavity, oropharynx, hypopharynx, and larynx. Patients with HNSCC have benefited greatly from the latest advances in surgical techniques, radiation therapy and chemotherapy. However, despite advances in local control and overall quality-of-life achieved with the use of combined modality therapies, the survival rates for $\mathrm{HNSCC}$ have only marginally improved over the past two decades [1]. Hence, novel methods of cancer detection and prognosis need to be developed.

HNSCC is a heterogeneous disease involving deregulation of multiple pathways linked to cellular differentiation, cell cycle control, apoptosis, angiogenesis, and metastasis [2]. The PI3K/AKT/mTOR signaling pathway has emerged as one of the most frequently altered in multiple cancers including HNSCC [3-5] and multiple upstream and downstream components such as Epidermal Growth Factor Receptor (EGFR), phosphatidylinositol 3-kinase (PI3K), protein kinase B (AKT/PKB), phosphatase and tensin homolog (PTEN) and mammalian target of rapamycin (mTOR) have been found deregulated, making this pathway very attractive for the development of molecular-targeted therapies [6].

The PI3K pathway has been found genetically deregulated in human cancers at various levels. The first identified genetic mechanism of PI3K pathway activation was the loss of PTEN function by mutation or deletion, leading to the accumulation of the PI3K product phosphatidylinositol $(3,4,5)$-trisphosphate $\left(\mathrm{PIP}_{3}\right)$. The accumulation of $\mathrm{PIP}_{3}$ activates a signaling cascade starting with the phosphorylation (activation) of the protein serinethreonine kinase AKT by 3-phosphoinositide dependent protein kinase-1 (PDK1/PDPK1). PDK1 has been considered a "master kinase" that phosphorylates and is responsible for the activation of other ACG kinase family members, many of them related to cell proliferation, survival or the inhibition of apoptosis, including AKT1, 2 and 3 [7].

Recent studies have reported high frequencies of somatic mutations in the PI3K catalytic subunit, $\mathrm{p} 110 \alpha$ gene $(P I K 3 C A)$, in various cancer types including HNSCC $[8,9]$. The majority of PIK3CA mutations have been clustered within the helical (exon 9) and catalytic (exon 20) protein domains [8]. Furthermore, three hotspot mutations have been identified: E542K, E545K (both mapping in exon 9) and H1047R (in exon 20), which have been shown to increase PI3K oncogenic activity and to confer transforming properties in vitro and in vivo [10]. The PIK3CA mutation rates reported in HNSCC studies range from $2.6 \%$ to $19 \%$ [5], found to be particularly common in HPV-positive oropharyngeal tumors (reaching $24-28 \%)$ [11].
Activating AKT1 mutations have also been described in solid tumors $[12,13]$. The E17K mutation in the Pleckstrin homology (PH) domain of the AKT1 gene can result in PI3K-independent membrane recruitment of AKT, recapitulating the effects of the AKT8 murine leukemia retrovirus GAG-AKT fusion protein. E17KAKT1 exhibits transforming activity in vitro and in vivo, albeit at lower level than the myristoylated AKT [13]. A case of $A K T 1$ mutation has been recently documented in a patient with high-risk HNSCC [14].

Nevertheless, the prognostic significance of PI3K/ AKT/mTOR pathway alterations and their impact on HNSCC patient survival remains to be established. This prompted us to perform a thorough analysis of $\mathrm{PI} 3 \mathrm{~K} / \mathrm{AKT} / \mathrm{mTOR}$ pathway components to investigate the mutational status of PIK3CA and AKT1 genes and the immunohistochemical expression of a number of pathway-related proteins (EGFR, PDK1, p-AKT, PTEN, p-S6) using a consecutive series of 93 surgically treated HNSCC patients, and to establish correlations with the clinicopathological parameters and disease outcome. The $\mathrm{PI} 3 \mathrm{~K} / \mathrm{AKT} / \mathrm{mTOR}$ pathway contains numerous putative therapeutic targets; however, to determine the prevalence and clinical relevance of molecular alterations in this pathway is fundamental to identify those with potential prognostic and therapeutic application to HNSCC patients.

\section{RESULTS}

\section{Significance of $P I K 3 C A$ and $A K T 1$ mutations in HNSCC patients}

The presence of the three activating hot-spot mutations (E542K, E545K and H1047R) in exons 9 and 20 of the PIK3CA gene and the oncogenic E17K mutation in the $\mathrm{PH}$ domain of the $A K T 1$ gene was analyzed in laryngeal and hypopharyngeal squamous cell carcinoma tissue specimens by mutant-enriched sequencing (Supplementary Figure S1). PIK3CA mutations were detected in 10 cases $(13 \%)$; nine cases harboring only c. $3140 \mathrm{~A}>\mathrm{G}(\mathrm{H} 1047 \mathrm{R})$ mutation and one case harboring both $\mathrm{H} 1047 \mathrm{R}$ and c.1624G $>\mathrm{A}(\mathrm{E} 542 \mathrm{~K})$. The $A K T 1$ c. $49 \mathrm{G}>\mathrm{A}(\mathrm{E} 17 \mathrm{~K})$ mutation was detected in seven cases (9\%) and in two cases AKT1 mutation coexisted with PIK3CA (H1047R) mutation.

The relationships between PIK3CA c. $3140 \mathrm{~A}>\mathrm{G}$ (H1047R) mutation, $A K T 1$ c.49G $>$ A (E17K) mutation and the clinicopathological variables are shown in Supplementary Table S1. PIK3CA (H1047R) mutation was more frequent in laryngeal carcinomas $(20 \%, 8$ cases in larynx vs 6\%, 2 cases in hypopharynx) and was significantly associated with stage I-II tumors $(P=0.002)$, and negative lymph node metastasis $(P=0.003)$.

In addition, PIK3CA (H1047R) mutation was more frequently detected in non-recurrent tumors (5 out of 6 cases; $P=0.077$ ) and only 1 tumor with local 
recurrence, whereas tumors with regional recurrence or distant metastasis did not harbor PIK3CA (H1047R) mutation. Univariate Kaplan-Meier analysis showed that patients with PIK3CA (H1047R) mutation experienced a better disease-specific survival almost reaching statistical significance (Log-rank test, $P=0.063$ ). Due to the complexity of our mutant-enriched sequencing methodology for routine clinical analysis, we also assessed the presence of PIK3CA mutations with two different kits used for diagnostic purposes in clinical laboratories (i.e. cobas ${ }^{\circledR} 4800$ PIK3CA Mutation Test by Roche Molecular Diagnostics and PIK3CA Mutation Analysis Kit by EntroGen). While results obtained with both diagnostic kits perfectly matched, $P I K 3 C A$ mutations were detected at a much lower frequency (1 positive case, $2 \%$ ) than using the mutant-enriched sequencing methods (10 positive cases, $13 \%$ ).

On the other hand, the presence of $A K T 1$ (E17K) mutation in this HNSCC series did not show influence on prognosis (Supplementary Table S1) or patient survival (Log-rank test, $P=0.427$ ).

\section{S6 phosphorylation is a common feature in HNSCC patients that correlates significantly with improved clinical outcome in laryngeal carcinomas}

The activation status of the PI3K/AKT/mTOR pathway was investigated in our series of 93 laryngeal and hypopharyngeal carcinomas by analyzing the immunohistochemical expression of multiple upstream and downstream components, such as EGFR, PDK1, PTEN, p-AKT and p-S6. Positive EGFR expression was found in 16\% (12/75) cases (Figure 1A and 1B) and showed no correlation with clinical parameters. Positive PDK1 expression was detected in $26(35 \%)$ out of 75 patients (Figure 1C and 1D). PDK1 expression did not correlate significantly with any of the clinicopathological features. Loss of PTEN expression was found in $40 \%$ $(30 / 75)$ tumors (Figure 1E and 1F). PTEN loss tended to correlate inversely with lymph node involvement in this cohort (Fisher's Exact test; $P=0.081$ ). Since PTEN is an inhibitor of the PI3K/AKT/mTOR pathway, this supports an association of PTEN loss with favorable prognosis.

We also analyzed the phosphorylation status of AKT at Ser473 and ribosomal protein S6 at both Ser235/236 and Ser240/244. Positive p-AKT expression was observed in $45 \%(34 / 75)$ cases (Figure $1 \mathrm{G}$ and $1 \mathrm{H})$; however, no correlations with clinical data were found. Activation of ribosomal protein S6 through phosphorylation on either Serine $235 / 236$ or Serine 240/244 was detected in $68 \%$ $(51 / 75)$ and $77 \%$ (58/75) tumors, respectively (Figure 1I-L). More importantly, positive p-S6 expression (in particular Ser235/236) was significantly correlated with clinicopathological parameters associated with better prognosis (Table 1). Thus, p-S6 (Ser235/236) was significantly associated with negative lymph node metastasis (Fisher's Exact test; $P<0.001$ ); smaller tumor size $(P=0.013)$; and lower disease stages $(P=0.001)$. Moreover, patients showing S6 phosphorylation on Serine $235 / 236$ exhibited lower risk of tumor recurrence $(P=$ $0.006)$, and significantly less distant metastasis $(P=0.003)$ and tumor-associated death $(P=0.002)$.

Furthermore, a multivariate regression model was constructed in order to evaluate the influence of the following molecular and clinicopathological parameters on lymph node involvement in an independent fashion: tumor size, localization, pathological grade, PTEN expression, PDK1 expression, PIK3CA (H1047R) mutation and S6 phosphorylation. Hypopharyngeal localization, positive PTEN expression, absence of PIK3CA (H1047R) mutation, and negative p-S6 (Ser235/236) showed an independent predictive value for lymph node metastasis. According to these data, the risk for nodal metastasis was estimated to be 5.8-fold higher in patients with hypopharyngeal tumors ( $\mathrm{HR}=5.82$; CI 95\% 1.32-25.64; $P=0.02), 4.7$-fold higher in patients with positive PTEN expression $(\mathrm{HR}=4.7$; CI 95\% 1.17-18.78; $P=0.028)$, 13.65-fold higher in patients without PIK3CA (H1047R) mutation $(\mathrm{HR}=13.65$; CI 95\% 1.36-137.38; $P=0.026)$, and remarkably 49.6-fold higher in patients with negative p-S6 (Ser235/236) tumors $(\mathrm{HR}=49.61$; CI 95\% 3.43$716.11 ; P=0.004)$.

Interestingly, using analogous multivariate models we found that S6 phosphorylation (Ser235/236) was the only significant independent predictor for distant metastasis. Thus, patients with negative p-S6 (Ser235/236) tumors exhibited 7.6-fold higher risk to develop distant metastasis than positive p-S6 (Ser235/236) tumors $(\mathrm{HR}=$ 7.66; CI 95\% 1.81-32.47; $P=0.006)$.

We next examined the influence of PI3K pathway components on patients' survival. Univariate KaplanMeier analysis showed that patients with tumors harboring S6 phosphorylation in either Ser235/236 or Ser240/244 had a significantly better disease-specific survival (Logrank test, $P<0.001$ and $P=0.002$ respectively, Figure $2 \mathrm{~A}$ and $2 \mathrm{~B}$ ). In addition, the results obtained from univariate Cox analysis are also shown in Table 2. Furthermore, multivariate Cox analysis was performed including tumor size, localization, lymph node metastasis, pathological grade, and p-S6 (Ser235/236) (Table 3). This model showed that only a negative $\mathrm{p}-\mathrm{S} 6$ (Ser235/236) expression was a significant independent predictor of reduced diseasespecific survival $(\mathrm{HR}=2.47$; CI 95\% 1.11-5.5; $P=0.026)$, and the presence of nodal metastasis was near significant $(\mathrm{HR}=3.1$; CI 95\% 0.97-9.89; $P=0.056)$.

To further confirm these data, immunohistochemical analysis of both p-S6 (Ser235/236) and (Ser240/244) was extended to a larger series of HNSCC patients including 432 additional cases from various tumor sites (oropharynx, larynx and hypopharynx). Analogous results were obtained in this extended patient cohort, confirming the relationship 
of S6 phosphorylation with clinicopathological parameters associated with a better prognosis (Supplementary Table S2) and improved disease-specific survival (Log-rank test, $P=0.003$ and $P=0.001$, Figure $2 \mathrm{C}$ and $2 \mathrm{D})$. The impact of p-S6 expression on disease course was also examined separately in each anatomic site, as we noted differences in p-S6 expression among the various tumor locations. Clear differences were observed on patient survival between the different subgroups. Specifically, we found that patients harboring laryngeal tumors with S6 phosphorylation on either Ser235/236 or Ser240/244 exhibited a significantly improved disease-specific survival (Log-rank test, $P=0.002$ and $P<0.001$, Figure $3 \mathrm{~A}$ and $3 \mathrm{~B})$, whereas differences did not reach statistical significance in the subgroups of hypopharyngeal and oropharyngeal carcinomas (Figure 3C-3F). Nevertheless, p-S6 expression also tended to associate with a better survival in these subgroups of patients. The results from Univariate Cox analysis in the laryngeal subgroup also showed that both p-S6 (Ser235/236) and (Ser240/244) were strongly and significantly associated with improved disease-specific survival and overall survival (Table 4), although p-S6 expression was not found to be a significant independent predictor in multivariate analysis $(\mathrm{HR}=1.24$; CI 95\% 0.65-2.32; $P=0.51$ for Ser235/236, and HR = 1.04; CI 95\% 0.45-2.38; $P=0.93$ for Ser240/244).

Together these data confirm the prevalence of S6 phosphorylation and consequently $\mathrm{mTORC} 1$ activation in HNSCC tissue specimens and also its clinical relevance on patient prognosis and disease outcome, particularly in the subgroup of laryngeal carcinoma patients.

\section{DISCUSSION}

Recent advances in deep sequencing have uncovered the complexity and heterogeneity of the HNSCC oncogenome [5, 15]. Despite the high diversity of genetic alterations underlying each individual tumor, most molecular alterations converge into few commonly deregulated pathways [16]. The PI3K/AKT/mTOR signaling pathway has emerged as the most frequently mutated mitogenic pathway in HNSCC (31\%) with multiple actionable targets, representing an excellent opportunity to develop more personalized therapeutic strategies [5, 16]. Nevertheless, the identification of clinically and biologically relevant features in this pathway is fundamental to define the central nodes that may be exploited therapeutically. Hence, using an unbiased cohort of consecutively and homogeneously treated (surgery) HNSCC patients, we conducted a comprehensive study to investigate the prognostic significance of multiple genetic and biochemical alterations in key players of the PI3K/AKT/mTOR pathway (i.e. PIK3CA and $A K T 1$ mutations and immunohistochemical expression of EGFR, PDK1, p-AKT, PTEN and p-S6). Collectively, our results suggest a positive correlation between the mTORC1 activation and a less aggressive phenotype, resulting in a significantly improved survival in the subgroup of laryngeal carcinomas.
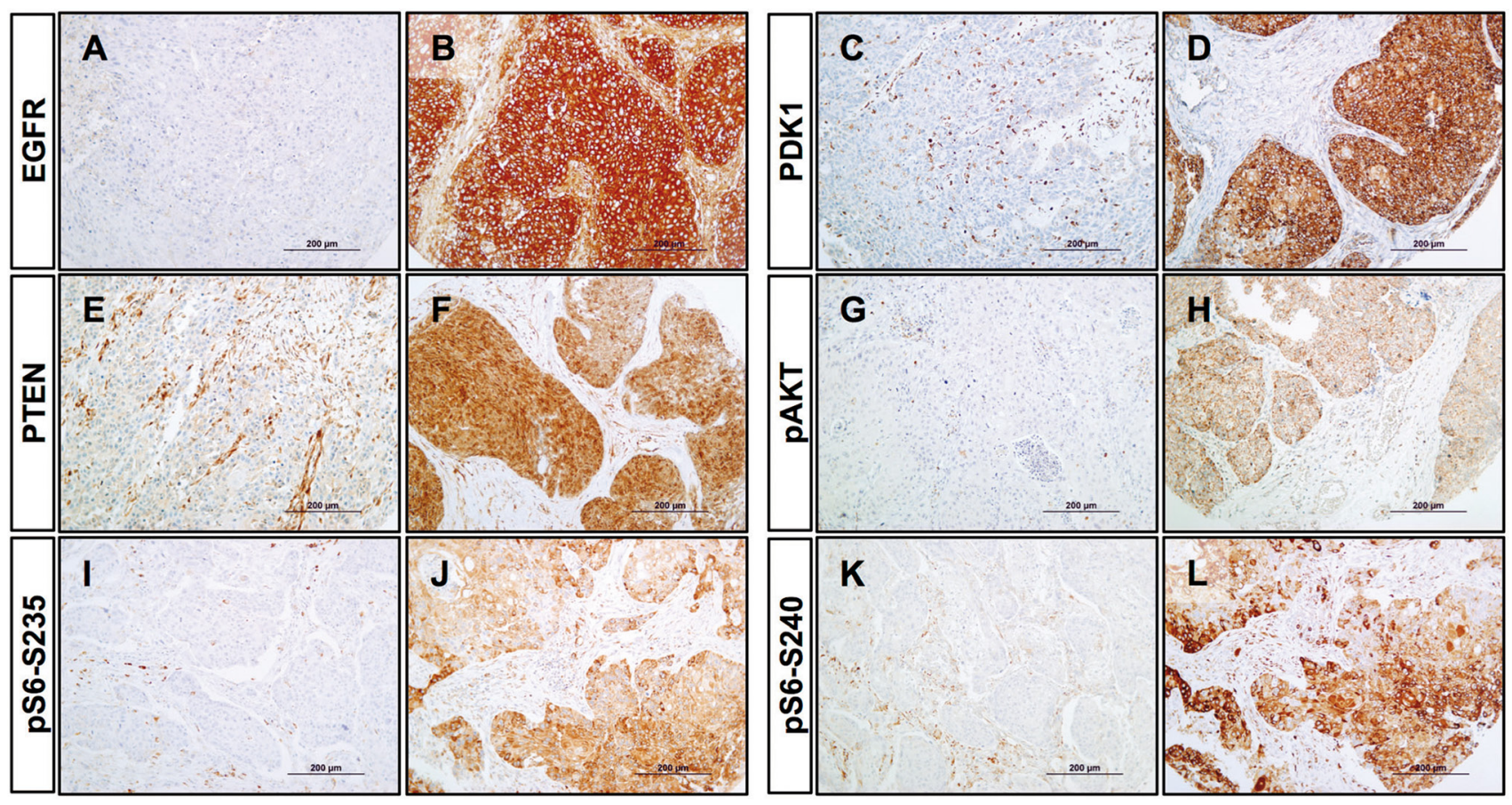

Figure 1: Immunohistochemical analysis of PI3K pathway proteins in HNSCC tissue specimens. Representative examples of negative and positive expression of EGFR A, B. PDK1 C, D. PTEN E, F. phosphorylation of AKT on Ser473 G, H. phosphorylation of S6 on Ser235/236 I, J. and phosphorylation of S6 on Ser240/244 K, L. Original magnification x200. 
Table 1: Associations of p-S6 protein expression with clinicopathological findings, relapse and disease outcome

\begin{tabular}{|c|c|c|c|c|c|}
\hline Characteristic & No. & $\begin{array}{c}\text { p-S6(Ser235) } \\
\text { expression (\%) }\end{array}$ & $\boldsymbol{P}^{\dagger}$ & $\begin{array}{c}\text { p-S6(Ser } 240) \\
\text { expression }(\%)\end{array}$ & $\boldsymbol{P}^{\dagger}$ \\
\hline \multicolumn{6}{|l|}{ - pT classification } \\
\hline $\mathrm{T} 1-\mathrm{T} 2$ & 29 & $25(86)$ & 0.013 & $26(90)$ & 0.118 \\
\hline $\mathrm{T} 3$ & 25 & $16(64)$ & & $18(72)$ & \\
\hline $\mathrm{T} 4$ & 21 & $10(48)$ & & $14(67)$ & \\
\hline \multicolumn{6}{|l|}{ - pN classification } \\
\hline N0 & 27 & $26(96)$ & $<0.001$ & $25(93)$ & 0.022 \\
\hline $\mathrm{N} 1-3$ & 48 & $25(52)$ & & $33(69)$ & \\
\hline \multicolumn{6}{|l|}{ - Disease stage } \\
\hline I-II & 15 & $15(100)$ & 0.001 & $15(100)$ & 0.023 \\
\hline III & 14 & $12(86)$ & & $12(86)$ & \\
\hline IV & 46 & $24(52)$ & & $31(67)$ & \\
\hline \multicolumn{6}{|l|}{ - Pathological grade } \\
\hline Well differentiated & 28 & $22(79)$ & 0.308 & $25(89)$ & 0.161 \\
\hline $\begin{array}{l}\text { Moderately } \\
\text { differentiated }\end{array}$ & 33 & $20(61)$ & & $23(70)$ & \\
\hline Poorly differentiated & 14 & $9(64)$ & & $10(71)$ & \\
\hline \multicolumn{6}{|l|}{ - Site } \\
\hline Hypopharynx & 34 & $20(59)$ & 0.142 & $23(68)$ & 0.097 \\
\hline Larynx & 41 & $31(76)$ & & $35(85)$ & \\
\hline $\begin{array}{l}\text { - Tumor Recurrence } \\
\text { (at five years) } \$\end{array}$ & 25 & $21(84)$ & 0.006 & $21(84)$ & 0.085 \\
\hline No & 32 & $15(47)$ & & $20(63)$ & \\
\hline \multicolumn{6}{|l|}{ Yes } \\
\hline \multicolumn{6}{|l|}{$\begin{array}{l}\text { - Disease status (at } \\
\text { five years) }\end{array}$} \\
\hline $\begin{array}{l}\text { Alive without } \\
\text { disease }\end{array}$ & 27 & $23(85)$ & 0.002 & $23(85)$ & 0.043 \\
\hline $\begin{array}{l}\text { Dead of index } \\
\text { cancer }\end{array}$ & 30 & $13(43)$ & & $18(60)$ & \\
\hline \multicolumn{6}{|l|}{ - } \\
\hline Died of other causes & 18 & $15(83)$ & & $17(94)$ & \\
\hline Total Cases & 75 & $51(68)$ & & $58(77)$ & \\
\hline
\end{tabular}

${ }^{\dagger}$ Fisher's exact test.

$\$$ Patients who died from causes not related to the index tumor were excluded from the recurrence analysis.

Remarkably, our findings reveal the high prevalence of S6 phosphorylation, a surrogate marker of mTORC1 activation, in HNSCC specimens $(>70 \%)$ and, more importantly, demonstrate for the first time its relevance on clinical outcome: phosphorylation of ribosomal protein S6 on either Ser235/236 or Ser240/244 was a significant inverse predictor of lymph node metastasis, regional recurrence and distant metastasis. Consequently, S6 phosphorylation was also correlated with improved disease-specific survival in these patients, although the differences were only significant in the subgroup of laryngeal carcinomas. Hence, measurement of S6 
phosphorylation by immunohistochemistry or by other means may help to stratify patients and/or to guide treatment decisions.

Activating PIK3CA (H1047R) mutation was the most frequently found mutation in our series $(13 \%$, 10 cases $)$, particularly frequent in the larynx $(20 \%, 8$ cases). Moreover, PIK3CA (H1047R) mutation was associated with a better clinical outcome in laryngeal and hypopharyngeal carcinoma patients. Since most of these mutations $(8 / 10)$ were present in laryngeal cancers, this association could be attributable to this location. In line with this, a recent analysis of TCGA sequencing data showed that $P I K 3 C A$ mutations tended to associate with improved survival in HNSCC patients [17].

Numerous evidences have associated activating PIK3CA mutations with a more tumorigenic phenotype $[18,19]$; however, contradicting results have been obtained when evaluating the prognostic significance of PIK3CA mutations in different human cancers. Thus, an association with favorable prognosis has been demonstrated in patients with breast [20] and esophageal carcinomas [21], whereas a poor prognosis has been reported in lung, colon and rectum cancer $[19,22,23]$.

Nevertheless, PIK3CA (H1047R) mutation rate observed in our series by mutant-enriched sequencing could not be confirmed by PIK3CA mutation analysis with two different kits routinely used for diagnostic purposes. Thus, PIK3CA mutations were detected at a much lower frequency ( 1 positive case, $2 \%$ ) than using the mutant-enriched sequencing methods (10 positive cases, $13 \%$ ). These differences may be attributable to a higher sensitivity of the mutant-enriched method (approximately $0.25 \%$ ), as we previously reported [24], questioning the prognostic relevance of these data, due to the low mutation rate observed at the sensitivity level (1-5\%) of current routine diagnostic methods.

These results clearly indicate that despite activating mutations may be partially contributing, they are not a major driving force for the frequent PI3K/AKT/mTOR pathway activation detected in HNSCC. Although the PI3K pathway has been reported to harbor the highest percentage of mutations (30.5\%) [5], widespread genomic
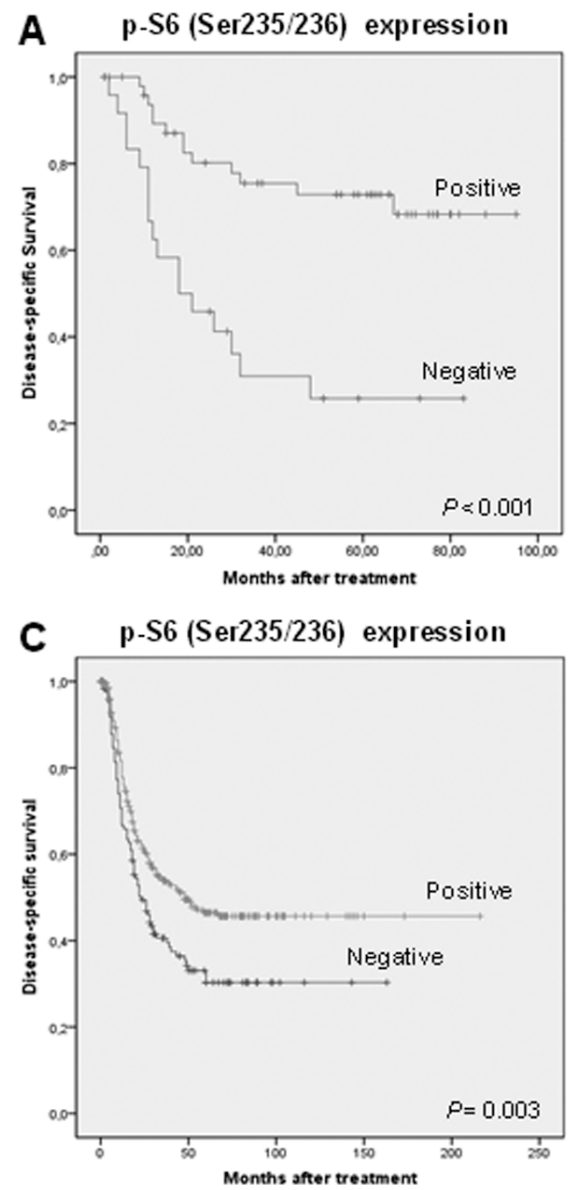
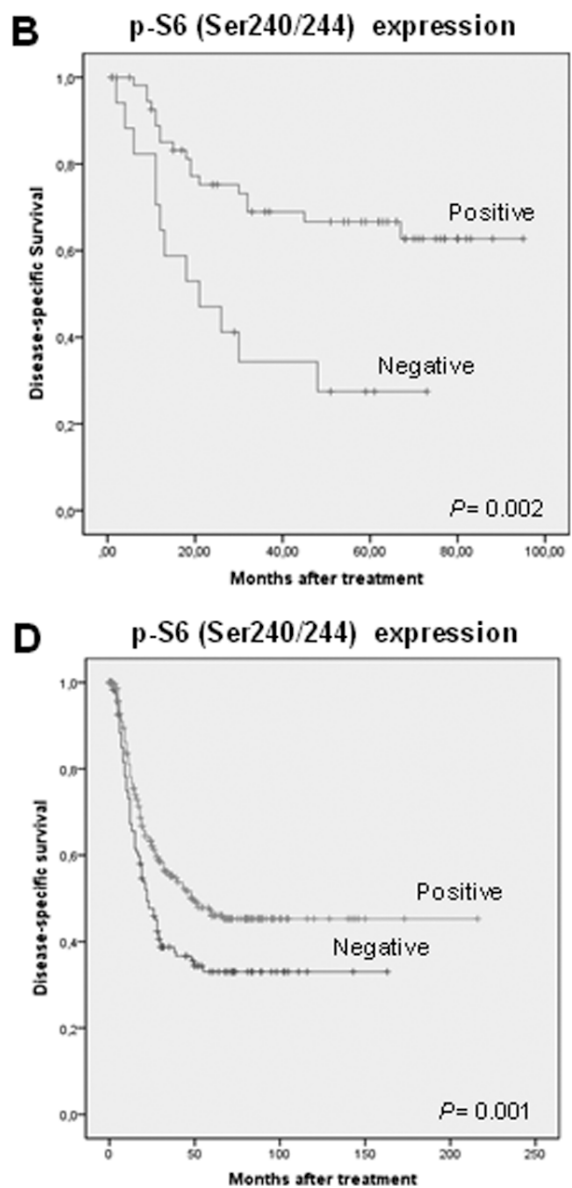

Figure 2: mTOR activation is associated with favorable prognosis in HNSCC patients. Kaplan-Meier disease-specific survival curves of the 93 patients included in the study categorized by S6 phosphorylation on Ser235/236 A. and S6 phosphorylation on Ser240/244 B. Kaplan-Meier disease-specific survival for the extended series of 432 HNSCC patients categorized by S6 phosphorylation on S235/236 C. and S6 phosphorylation on Ser240/244 D. $P$ values were estimated using the log-rank test. 
Table 2: Univariate Cox regression analysis for the disease-free survival (DSS) and the overall survival (OS)

\begin{tabular}{|c|c|c|c|c|c|c|}
\hline Parameter & 5-year DSS & $\begin{array}{c}\text { HR } \\
(95 \% \mathrm{CI}) \\
\end{array}$ & $P$ & 5-year OS & HR $(95 \% C I)$ & $P$ \\
\hline \multicolumn{7}{|l|}{ Localization } \\
\hline Larynx & $70 \%$ & 1 & & $56 \%$ & 1 & \\
\hline Hypopharynx & $41 \%$ & $\begin{array}{c}2.19 \\
(1.06- \\
4.55)\end{array}$ & 0.035 & $38 \%$ & $1.8(0.96-3.36)$ & 0.06 \\
\hline \multicolumn{7}{|l|}{ T classification } \\
\hline T1-T2 & $68 \%$ & 1 & & $54 \%$ & 1 & \\
\hline T3-T4 & $50 \%$ & $\begin{array}{c}1.63 \\
(0.74- \\
3.55)\end{array}$ & 0.21 & $44 \%$ & $1.2(0.63-2.26)$ & 0.59 \\
\hline \multicolumn{7}{|l|}{$\mathbf{N}$ classification } \\
\hline N0 & $87 \%$ & 1 & & $69 \%$ & 1 & \\
\hline N1-3 & $41 \%$ & $\begin{array}{c}4.89(1.7- \\
14.1)\end{array}$ & 0.003 & $35 \%$ & $2.7(1.26-5.64)$ & 0.01 \\
\hline \multicolumn{7}{|l|}{$\begin{array}{l}\text { Pathological } \\
\text { grade }\end{array}$} \\
\hline G1-G2 & $62 \%$ & 1 & & $52 \%$ & 1 & \\
\hline G3 & $35 \%$ & $\begin{array}{c}2.02 \\
(0.89- \\
4.55)\end{array}$ & 0.11 & $31 \%$ & $1.7(0.69-3.5)$ & 0.18 \\
\hline \multicolumn{7}{|l|}{$\begin{array}{l}\text { p-S6 } \\
(\text { Ser235/236) }\end{array}$} \\
\hline Positive & $73 \%$ & 1 & & $60 \%$ & 1 & \\
\hline Negative & $26 \%$ & $\begin{array}{c}3.93 \\
(1.89- \\
8.16)\end{array}$ & $<0.001$ & $21 \%$ & $2.6(1.38-4.89)$ & 0.004 \\
\hline \multicolumn{7}{|l|}{$\begin{array}{l}\text { p-S6 } \\
\text { (Ser240/244) }\end{array}$} \\
\hline Positive & $67 \%$ & 1 & & $54 \%$ & 1 & \\
\hline Negative & $26 \%$ & $\begin{array}{c}2.96 \\
(1.41- \\
6.19)\end{array}$ & 0.006 & $27 \%$ & $2(1.01-3.99)$ & 0.045 \\
\hline
\end{tabular}

HR Hazard Ratio, CI Confidence Interval

and epigenetic alterations may account for the frequent activation of the PI3K/AKT/mTOR axis in HNSCC. Various plausible mechanisms have been thoroughly discussed by Iglesias-Bartolome et al. [16]. In addition to activating PIK3CA mutations, copy number gains and mRNA overexpression are also frequent events in HNSCC (at $20 \%$ and $52 \%$, respectively). In a previous study, we detected PIK3CA gene amplification in $37 \%$ fresh HNSCC samples, and $A K T 2$ gene amplification in $30 \%$ tumors [3]. In addition, the present study also showed frequent PTEN loss and p-AKT in $40 \%$ and $45 \%$ cases respectively, which all may result in the activation of downstream targets such as mTOR. It is also worth noting that none of these upstream components individually or combined had an impact on patient survival in our series. Consequently, they are unlikely to be responsible for the good prognosis associated to the sustained mTORC1 activation, as reflected by $\mathrm{S} 6$ phosphorylation. Alternatively, it has also been demonstrated that there is a crosstalk between PI3K/ AKT/mTOR pathway and the RAS/RAF/MEK/ERK 
Table 3: Multivariate Cox regression analysis for the disease-free survival (DSS) and the overall survival (OS)

\begin{tabular}{|c|c|c|c|c|}
\hline Parameter & HR (95\% CI) DSS & $P$ & HR $(95 \% \mathrm{CI}) \mathrm{OS}$ & $P$ \\
\hline \multicolumn{5}{|l|}{ Localization } \\
\hline Larynx & 1 & & 1 & \\
\hline Hypopharynx & $1.19(0.54-2.65)$ & 0.66 & $1.16(0.58-2.35)$ & 0.67 \\
\hline \multicolumn{5}{|l|}{$T$ classification } \\
\hline $\mathrm{T} 1-\mathrm{T} 2$ & 1 & & 1 & \\
\hline $\mathrm{T} 3-\mathrm{T} 4$ & $1.07(0.48-2.38)$ & 0.88 & $1.17(0.6-2.28)$ & 0.64 \\
\hline \multicolumn{5}{|l|}{$\mathbf{N}$ classification } \\
\hline N0 & 1 & & 1 & \\
\hline N1-3 & $3.01(0.97-9.89)$ & 0.056 & $1.99(0.86-4.59)$ & 0.11 \\
\hline \multicolumn{5}{|c|}{ Pathological grade } \\
\hline G1-G2 & 1 & & 1 & \\
\hline G3 & $1.61(0.71-3.69)$ & 0.25 & $1,42(0.67-3.03)$ & 0.36 \\
\hline \multicolumn{5}{|c|}{ p-S6 (Ser235/236) } \\
\hline Positive & 1 & & 1 & \\
\hline Negative & $2.47(1.11-5.5)$ & 0.026 & $2.6(1.38-4.89)$ & 0.003 \\
\hline
\end{tabular}

HR Hazard Ratio, CI Confidence Interval

pathway in cancers [25]. Furthermore, another interesting possibility of specific relevance to HNSCC is that REDD1, SESTRIN1 and SESTRIN2, all downstream targets of p53 and negative regulators of mTOR, are presumably disabled in more than $50 \%$ of HNSCC harboring TP53 mutations what could result in mTOR activation in the absence of any other obvious genomic alteration of the PI3K pathway.

Various pathway activation phenotypes have been described in cancer and normal cells that could potentially explain the association with improved prognosis, including oncogene-induced senescence, maintenance of differentiation, and anti-invasive/anti-metastatic phenotypes [26-28]. Concordant with our in vivo data on HNSCC patients, these observations strongly and consistently reinforce that mTORC1 activation in the context of HNSCC may elicit anti-invasive and antimetastatic phenotypes in a subset of patients.

mTOR activity has been associated to increased cell motility and metastasis in colorectal cancer, prostate cancer and lymphangioleiomyomatosis (LAM)[29, 30]. In addition, mTOR inhibition has been found to either decrease or promote cell migration and invasion depending on the cell system [29, 31, 32], thus indicating that mTOR activation only results in increased metastatic capability in a very specific cellular context. The fact that HNSCCs with mTORC1 activation (p-S6-positive) have a comparatively better survival than those with less active
mTORC1, does not preclude the potential therapeutic value of PI3K/mTOR inhibitors for HNSCCs. In fact, extensive work has been performed defining the pathway may represent a viable molecular target in this tumor type. Rapamycin treatment has been used in different oncogenic HNSCCs models and seems to constitute a therapeutic alternative, for at least a subset of patients [16, 33-35]. In addition, multiple PI3K/AKT/mTOR inhibitors have been developed targeting different nodes of this pathway, mainly PI3K isoforms, AKT, mTORC1 and /or mTORC2 complexes, which are currently into clinical trials. In future work, it would be of interest to investigate if HNSCCs with p-S6-positive phenotype are more sensitive to PI3K/mTOR inhibitors than p-S6-negative tumors.

The clinical impact of hyperactive mTOR depends on the tumor type as it has been revealed in two recent meta-analyses examining data from numerous previous studies [36, 37]. The combined message of these metaanalyses is that hyperactive mTOR associates with worse prognosis in gynecological and gastrointestinal cancers; it is not associated with worse prognosis in breast cancer or even associated with good prognosis in some breast cancer types [38] or in lung cancer [36]; and it is inconclusive (due to small sample sizes) in HNSCCs and prostate cancers. Our results provide further evidence showing that mTOR activation (by means of $\mathrm{p}-\mathrm{S} 6$ expression) has a distinct impact on patient survival depending on the tumor localization. 
In relation to the subsite-specific effect of pS6, although we cannot provide a precise explanation on the underlying mechanism of this differential effect, remarkably, analogous observations have been reported for other molecular alterations in a recent analysis of HNSCC genomic alterations using TCGA data [17], and also in previous studies from our group [39, 40]. Thus, a number of molecular alterations (such as Cortactin, Anoctamin 1, EGFR amplification or even PIK3CA mutation) have been shown to have a differential impact on patient survival specifically in the larynx. Therefore, this underscores that, in addition to molecular alterations, local anatomical circumstances also play a role in patient outcome. Further investigation into the biologic differences between larynx and other subsites should be performed.
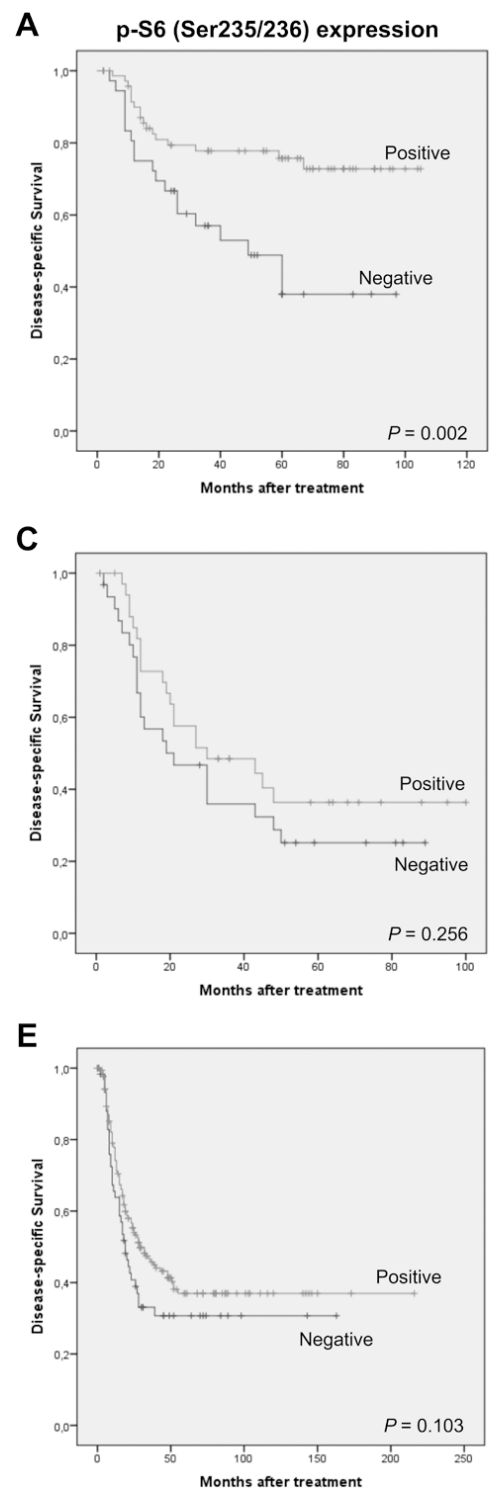

To summarize, our study unveils an unprecedented correlation between mTOR activation and favorable prognosis and improved clinical outcome in laryngeal carcinoma patients, and uncovers the potential of S6 phosphorylation as a good prognostic biomarker and an inverse predictor of lymph node and distant metastases. Activation of the PI3K/AKT/mTOR pathway is considered to play a crucial role in human neoplasms and so numerous drugs targeting this pathway have been developed and are currently in clinical trials [6, 41-43] including HNSCC (reviewed in [44]. Therefore, our results should be of broad interest, as immunohistochemical detection of p-S6 may help to stratify patients and guide treatment decisions.
B

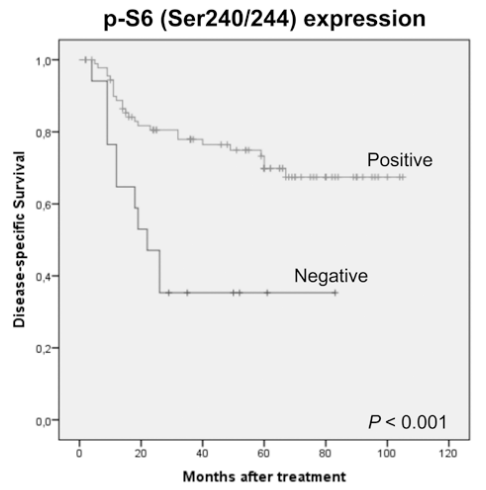

D

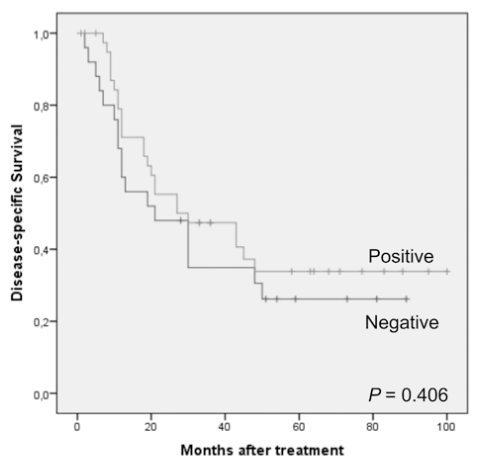

$\mathbf{F}$

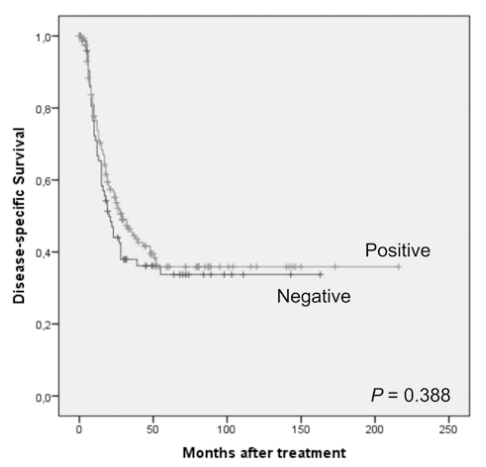

Figure 3: Distinctive effect of S6 phosphorylation on patients' survival depending on the tumor site. Kaplan-Meier disease-specific survival curves categorized by S6 phosphorylation on Ser235/236 and S6 phosphorylation on Ser240/244 on the subgroup of laryngeal A, B. hypopharyngeal C, D. and oropharyngeal carcinomas $\mathbf{E}, \mathbf{F}$. 
Table 4: Univariate Cox regression analysis for the disease-free survival (DSS) and the overall survival (OS) in the laryngeal subgroup of patients

\begin{tabular}{|c|c|c|c|c|c|c|}
\hline Parameter & 5-year DSS & $\begin{array}{c}\text { HR } \\
(95 \% \mathrm{CI})\end{array}$ & $P$ & 5-year OS & HR $(95 \% C I)$ & $P$ \\
\hline \multicolumn{7}{|c|}{ T classification } \\
\hline $\mathrm{T} 1-\mathrm{T} 2$ & $88 \%$ & 1 & & $72 \%$ & 1 & \\
\hline T3-T4 & $51 \%$ & $\begin{array}{c}4.47 \\
(1.85- \\
10.81)\end{array}$ & 0.21 & $38 \%$ & $2.52(1.35-4.59)$ & 0.003 \\
\hline \multicolumn{7}{|c|}{$\mathbf{N}$ classification } \\
\hline No & $85 \%$ & 1 & & $72 \%$ & 1 & \\
\hline $\mathrm{N} 1-3$ & $33 \%$ & $\begin{array}{c}6.53 \\
(3.03- \\
14.08)\end{array}$ & $<0.001$ & $28 \%$ & $4.18(2.29-7.42)$ & $<0.001$ \\
\hline \multicolumn{7}{|c|}{$\begin{array}{l}\text { Pathological } \\
\text { grade }\end{array}$} \\
\hline G1-G2 & $70 \%$ & 1 & & $59 \%$ & 1 & \\
\hline G3 & $31 \%$ & $\begin{array}{c}3.47 \\
(1.68- \\
7.16)\end{array}$ & 0.001 & $19 \%$ & $2.74(1.44-5.2)$ & 0.002 \\
\hline \multicolumn{7}{|c|}{$\begin{array}{l}\text { p-S6 } \\
\text { (Ser235/236) }\end{array}$} \\
\hline Positive & $76 \%$ & 1 & & $64 \%$ & 1 & \\
\hline Negative & $38 \%$ & $\begin{array}{c}2.67 \\
(1.38- \\
5.17)\end{array}$ & 0.003 & $28 \%$ & $2.27(1.31-3.93)$ & 0.003 \\
\hline \multicolumn{7}{|c|}{$\begin{array}{l}\text { p-S6 } \\
\text { (Ser240/244) }\end{array}$} \\
\hline Positive & $70 \%$ & 1 & & $61 \%$ & 1 & \\
\hline Negative & $35 \%$ & $\begin{array}{c}3.34 \\
(1.62- \\
6.87)\end{array}$ & 0.001 & $25 \%$ & $2.72(1.43-5.16)$ & 0.002 \\
\hline
\end{tabular}

HR Hazard Ratio, CI Confidence Interval

\section{MATERIALS AND METHODS}

\section{Patients and tissue specimens}

Surgical tissue specimens from 93 patients with laryngeal and hypopharyngeal squamous cell carcinoma who underwent surgical treatment at the Hospital Universitario Central de Asturias were collected, in accordance to approved institutional review board guidelines. All experimental protocols were approved by the Institutional Ethics Committee of the Hospital Universitario Central de Asturias. Informed consent was obtained from all patients. Representative tissue sections were obtained from archival, formalin-fixed and paraffin- embedded (FFPE) blocks and the histological diagnosis was confirmed by an experienced pathologist (AA).

This series included all patients with laryngeal and hypopharyngeal carcinomas consecutively treated between 2002 and 2005, who met the following criteria: a single primary tumor, microscopically clear surgical margins, absence of distant metastasis at the time of diagnosis and no treatment prior to surgery. Only three patients were women, the mean age was 60 years (range 36 to 86 years). All but four patients (96\%) were habitual tobacco and alcohol consumers. $41(44 \%)$ patients received postoperative radiotherapy (this was administered to $\mathrm{pT} 4$ and/or pN2-N3 patients). The characteristics of the patients studied and the 
clinicopathological features of their tumors (site, pT classification, $\mathrm{pN}$ classification, disease stage, and pathological grade) are shown in Supplementary Table S1. The stage of disease was determined after the surgical resection of the tumor according to the TNM system of the International Union against Cancer (7th Edition). The histological grade was determined according to the degree of differentiation of the tumor (Broders' classification).

The median follow-up of the whole series was 33 months (range, 1-95 months), and the median follow-up of the patients alive at the last visit was 68 months (range, 51-95 months). Data on HPV status were available for the whole series, as previously described $[45,46]$. As HPV infection may be a confounding factor, two HPV-positive patients were excluded from all the analyses.

In addition, a large cohort of 432 homogeneous surgically treated HNSCC patients was selected as a validation series. This series included patients with laryngeal, oropharyngeal and hypopharyngeal carcinomas treated at the Hospital Universitario Central de Asturias between 1990 and 2009, who met the same inclusion criteria above-described for the exploratory series. All patients had a single primary tumor, microscopically clear surgical margins and received no treatment prior to surgery. Only fourteen patients were women, and the mean age was 59 years (range 33 to 86 years). All but twelve (97\%) patients were habitual tobacco smokers and 404 were alcohol drinkers. The characteristics of the patients studied and the clinicopathological features of their tumors are shown in Supplementary Table S2. 244 (56\%) of 432 patients received postoperative radiotherapy. Only HPVnegative patients were included in the analysis.

\section{Tissue microarray construction}

Five morphologically representative areas were selected from each individual paraffin-embedded tumor block: two for DNA isolation and three for the construction of a tissue microarray (TMA). To avoid cross-contamination, two punches of $2 \mathrm{~mm}$ diameter were taken first, using a new, sterile punch (Kai Europe $\mathrm{GmbH}$, Solingen, Germany) for every tissue block, and stored in Eppendorf tubes at room temperature to be used for DNA extraction. Subsequently, three $1 \mathrm{~mm}$ tissue cylinders were taken from each of the 93 HNSCC to construct TMA blocks, as described previously [47]. In addition, each TMA also contained three cores of normal epithelium as an internal control. In order to check the histopathologic diagnosis and the adequacy of tissue sampling, a section from each TMA was stained with hematoxylin and eosin and examined by light microscopy. The same procedure was employed to construct TMA blocks from all the HNSCC patients included in the validation cohort.

\section{DNA extraction and mutational analysis of $P I K 3 C A$ and $A K T 1$ genes}

FFPE tumor cylinders were firstly deparaffinized in xylene and rehydrated through graded ethanol solutions. Subsequently, the tissue pellets were digested in lysis buffer containing $2 \mu \mathrm{g} / \mu \mathrm{L}$ proteinase $\mathrm{K}$ and the genomic DNA isolated using the QIAamp DNA Mini Kit (Qiagen, Valencia, CA) following manufacturer's instructions.

Mutations in exons 9 and 20 of the PIK3CA gene and the E17K hot-spot mutation of the AKT1 gene were analyzed by direct genomic sequencing methods and confirmed by using mutant-enriched sequencing methods that we previously developed [48]. All PCR fragments were purified using ExoSAP-IT kit (Affymetrix, Santa Clara, CA) and sequencing was performed with ABI Prism 3730xl DNA analyzers by Genewiz, Inc (South Plainfield, NJ). Any alteration detected was further verified by sequencing of a second PCR product derived independently from the original DNA template.

PIK3CA mutations were also assessed using two different kits designed for diagnostic purposes i.e. cobas ${ }^{\circledR} 4800$ PIK3CA Mutation Test by Roche Molecular Diagnostics (Branchburg, NJ) and PIK3CA Mutation Analysis Kit by EntroGen (Los Angeles, CA), following manufacturer's protocols.

\section{Immunohistochemistry}

The HNSCC TMAs were cut into 3- $\mu \mathrm{m}$ sections and dried on Flex IHC microscope slides (Dako). The sections were deparaffinized with standard xylene and hydrated through graded alcohols into water. Antigen retrieval was performed using Envision Flex Target Retrieval solution, high $\mathrm{pH}$ (Dako) or with proteinase $\mathrm{K}$ (for EGFR). Staining was done at room temperature on an automatic staining workstation (Dako Autostainer Plus) using the Dako EnVision Flex + Visualization System (Dako Autostainer) and the following antibodies: EGFR pharmDx (TM) kit (Dako), mouse monoclonal anti-PKB kinase (PDK1) (E-3; Santa Cruz; sc-17765) at 1:2000 dilution, rabbit monoclonal anti-Human phospho-Akt (Ser473), Phosphorylation Site Specific, (Clone 14-5; Dako; M3628) at 1:20 dilution, rabbit anti-phospho-S6 Ribosomal Protein (Ser235/236) (Cell Signaling \# 2211) at 1:200 dilution, rabbit monoclonal Phospho-S6 Ribosomal Protein (Ser240/244) (D68F8 XP®; Cell Signaling \# $5364)$ at 1:200 dilution, and mouse monoclonal antiHuman PTEN (Clone 6H2.1; Dako; M3627) at 1:50 dilution. Counterstaining with hematoxylin was the final step.

The slides were viewed randomly, without clinical data, by two of the authors. The average intra- and interobserver variation was $<5 \%$. Since EGFR, PDK1 and PTEN staining showed a homogeneous distribution, a semi-quantitative scoring system based on staining 
intensity was applied. Thus, EGFR immunostaining was scored as negative (0), weak (1), moderate (2) and strong protein expression (3) and PDK1 and PTEN were scored as negative (0), weak (1), strong (2) staining. For statistical purposes, EGFR and PDK1 staining data were dichotomized as negative expression (scores 0 -1) versus positive expression (scores $>1$ ) and for PTEN score 0 was considered loss of PTEN expression.

Immunostaining for $\mathrm{p}-\mathrm{AKT}$ and $\mathrm{p}-\mathrm{S} 6$ was evaluated using a semi-quantitative scale $(0 \%,<10 \%, 10-50 \%$, or $>50 \%$ positive tumor cells). For statistical analysis, staining data were dichotomized as negative expression (0-10\% stained cells) versus positive expression $(>10 \%$ stained cells), previously established as p-AKT cut-off level [49].

\section{Statistical analyses}

All the statistical analyses were performed using complete data sets. The experimental results distributed among the different clinical groups of tumors were tested for significance employing the Fisher's exact test. Logistic regression models were constructed to evaluate the independent effect (Odds Ratio) of the molecular variables on a given clinicopathological feature. Survival curves were calculated using the Kaplan-Meier product limit estimate. Differences between survival times were analyzed by the log-rank method and the Hazard Ratio (with 95\% CI) was calculated by univariate Cox regression analysis. Multivariate Cox proportional hazard models were used in a stepwise manner, and each variable was tested with the other factors as a co-variable. The HR with $95 \% \mathrm{CI}$ and $P$ values were reported. All tests were two-sided. The values of $P \leq 0.05$ were considered statistically significant.

\section{ACKNOWLEDGMENTS}

We thank Aitana Vallina and Laura Alonso-Durán for excellent technical assistance, and Teresa Ortega Montoliu and OIB staff for administrative support.

\section{CONFLICTS OF INTEREST}

The authors declare no conflicts of interest.

\section{GRANT SUPPORT}

This study was supported by grants from the Plan Nacional de I+D+I 2008-2011 Instituto de Salud Carlos III (PI11/00929 to JPR) and Plan Nacional de I+D+I 20132016 ISCIII (CP13/00013 and PI13/00259 to JMGP), RD12/0036/0015 of Red Temática de Investigación Cooperativa en Cáncer (RTICC), Spain, and the FEDER Funding Program from the European Union. M.A.V. is recipient of a fellowship from ISCIII (CD13/00157). Work in the laboratory of G.S. was funded by grant NIH R01CA109525.

\section{REFERENCES}

1. Haddad RI, Shin DM. Recent advances in head and neck cancer. N. Engl. J. Med. 2008; 359: 1143-1154.

2. Leemans CR, Braakhuis BJ, Brakenhoff RH. The molecular biology of head and neck cancer. Nat. Rev. Cancer 2011; 11: 9-22.

3. Pedrero JM, Carracedo DG, Pinto CM, Zapatero AH, Rodrigo JP, Nieto CS, Gonzalez MV. Frequent genetic and biochemical alterations of the PI3-K/AKT/PTEN pathway in head and neck squamous cell carcinoma. Int. J. Cancer 2005; 114: 242-248.

4. Agrawal N, Frederick MJ, Pickering CR, Bettegowda C, Chang K, Li RJ, Fakhry C, Xie TX, Zhang J, Wang J, Zhang $\mathrm{N}$, El-Naggar AK, Jasser SA, et al. Exome sequencing of head and neck squamous cell carcinoma reveals inactivating mutations in NOTCH1. Science 2011; 333: 1154-1157.

5. Lui VW, Hedberg ML, Li H, Vangara BS, Pendleton K, Zeng Y, Lu Y, Zhang Q, Du Y, Gilbert BR, Freilino M, Sauerwein S, Peyser ND, et al. Frequent mutation of the PI3K pathway in head and neck cancer defines predictive biomarkers. Cancer Discov. 2013; 3: 761-769.

6. Courtney KD, Corcoran RB, Engelman JA. The PI3K pathway as drug target in human cancer. J. Clin. Oncol. 2010; 28: 1075-1083.

7. Bayascas JR. Dissecting the role of the 3-phosphoinositidedependent protein kinase-1 (PDK1) signalling pathways. Cell Cycle 2008; 7: 2978-2982.

8. Samuels Y, Wang Z, Bardelli A, Silliman N, Ptak J, Szabo S, Yan H, Gazdar A, Powell SM, Riggins GJ, Willson JK, Markowitz S, Kinzler KW, et al. High frequency of mutations of the PIK3CA gene in human cancers. Science 2004; 304: 554.

9. Qiu W, Schönleben F, Li X, Ho DJ, Close LG, Manolidis $\mathrm{S}$, Bennett BP, Su GH. PIK3CA mutations in head and neck squamous cell carcinoma. Clin. Cancer Res. 2006; 12: 1441-1446.

10. Bader AG, Kang S, Vogt PK. Cancer-specific mutations in PIK3CA are oncogenic in vivo. Proc. Natl. Acad. Sci. U S A 2006; 103: 1475-1479.

11. Sewell A, Brown B, Biktasova A, Mills GB, Lu Y, Tyson DR, Issaeva N, Yarbrough WG. Reverse-phase protein array profiling of oropharyngeal cancer and significance of PIK3CA mutations in HPV-associated head and neck cancer. Clin. Cancer Res. 2014; 20: 2300-2311.

12. Brugge J, Hung MC, Mills GB. A new mutational AKTivation in the PI3K pathway. Cancer Cell 2007; 12: 104-107.

13. Carpten JD, Faber AL, Horn C, Donoho GP, Briggs SL, Robbins CM, Hostetter G, Boguslawski S, Moses TY, Savage S, Uhlik M, Lin A, Du J, et al. A transforming mutation in the pleckstrin homology domain of AKT1 in cancer. Nature 2007; 448: 439-444. 
14. McBride SM, Rothenberg SM, Faquin WC, Chan AW, Clark JR, Ellisen LW, Wirth LJ. Mutation frequency in 15 common cancer genes in high-risk head and neck squamous cell carcinoma. Head Neck 2014; 36: 1181-1188.

15. Pickering CR, Zhang J, Yoo SY, Bengtsson L, Moorthy S, Neskey DM, Zhao M, Ortega Alves MV, Chang K, Drummond J, Cortez E, Xie TX, Zhang D, et al. Integrative genomic characterization of oral squamous cell carcinoma identifies frequent somatic drivers. Cancer Discov. 2013; 3: 770-781.

16. Iglesias-Bartolome R, Martin D, Gutkind JS. Exploiting the head and neck cancer oncogenome: widespread PI3KmTOR pathway alterations and novel molecular targets. Cancer Discov. 2013; 3: 722-725.

17. Birkeland AC, Ludwig ML, Meraj TS, Brenner JC, Prince ME. The Tip of the Iceberg: Clinical Implications of Genomic Sequencing Projects in Head and Neck Cancer. Cancers (Basel). 2015; 7: 2094-2109.

18. Samuels Y, Diaz LA Jr, Schmidt-Kittler O, Cummins JM, Delong L, Cheong I, Rago C, Huso DL, Lengauer C, Kinzler KW, Vogelstein B, Velculescu VE. Mutant PIK3CA promotes cell growth and invasion of human cancer cells. Cancer Cell 2005; 7: 561-573.

19. Ogino S, Nosho K, Kirkner GJ, Shima K, Irahara N, Kure S, Chan AT, Engelman JA, Kraft P, Cantley LC, Giovannucci EL, Fuchs CS. PIK3CA mutation is associated with poor prognosis among patients with curatively resected colon cancer. J. Clin. Oncol. 2009; 27: 1477-1484.

20. Kalinsky K, Jacks LM, Heguy A, Patil S, Drobnjak M, Bhanot UK, Hedvat CV, Traina TA, Solit D, Gerald W, Moynahan ME. PIK3CA mutation associates with improved outcome in breast cancer. Clin. Cancer Res. 2009; 15: 5049-5059.

21. Shigaki H, Baba Y, Watanabe M, Murata A, Ishimoto T, Iwatsuki M, Iwagami S, Nosho K, Baba H. PIK3CA mutation is associated with a favorable prognosis among patients with curatively resected esophageal squamous cell carcinoma. Clin. Cancer Res. 2013; 19: 2451-2459.

22. He Y, Van't Veer LJ, Mikolajewska-Hanclich I, van Velthuysen ML, Zeestraten EC, Nagtegaal ID, van de Velde CJ, Marijnen CA. PIK3CA mutations predict local recurrences in rectal cancer patients. Clin. Cancer Res. 2009; 15: 6956-6962.

23. De Roock W, Claes B, Bernasconi D, De Schutter J, Biesmans B, Fountzilas G, Kalogeras KT, Kotoula V, Papamichael D, Laurent-Puig P, Penault-Llorca F, Rougier P, Vincenzi B, et al. Effects of KRAS, BRAF, NRAS, and PIK3CA mutations on the efficacy of cetuximab plus chemotherapy in chemotherapy-refractory metastatic colorectal cancer: a retrospective consortium analysis. The Lancet Oncol. 2010; 11: 753-762.

24. Qiu W, Tong GX, Manolidis S, Close LG, Assaad AM, $\mathrm{Su}$ GH. Novel mutant-enriched sequencing identified high frequency of PIK3CA mutations in pharyngeal cancer. Int J Cancer. 2008; 122: 1189-1194.
25. Ventura AP, Radhakrishnan S, Green A, Rajaram SK, Allen AN, O'Briant K, Schummer M, Karlan B, Urban N, Tewari M, Drescher C, Knudsen BS. Activation of the MEK-S6 pathway in high-grade ovarian cancers. Appl Immunohistochem Mol Morphol. 2010; 18: 499-508.

26. Miyauchi H, Minamino T, Tateno K, Kunieda T, Toko H, Komuro I. Akt negatively regulates the in vitro lifespan of human endothelial cells via a $553 / \mathrm{p} 21$-dependent pathway. EMBO J. 2004; 23: 212-220.

27. Oyama K, Okawa T, Nakagawa H, Takaoka M, Andl CD, Kim SH, Klein-Szanto A, Diehl JA, Herlyn M, El-Deiry W, Rustgi AK. AKT induces senescence in primary esophageal epithelial cells but is permissive for differentiation as revealed in organotypic culture. Oncogene 2007; 26: 2353-2364.

28. Hutchinson JN, Jin J, Cardiff RD, Woodgett JR, Muller WJ. Activation of Akt-1 (PKB-alpha) can accelerate ErbB2-mediated mammary tumorigenesis but suppresses tumor invasion. Cancer Res. 2004; 64: 3171-3178.

29. Gulhati P, Bowen KA, Liu J, Stevens PD, Rychahou PG, Chen M, Lee EY, Weiss HL, O'Connor KL, Gao T, Evers BM. mTORC1 and mTORC2 regulate EMT, motility, and metastasis of colorectal cancer via RhoA and Rac1 signaling pathways. Cancer Res. 2011; 71: 3246-3256.

30. Barnes EA, Kenerson HL, Mak BC, Yeung RS. The loss of tuberin promotes cell invasion through the ss-catenin pathway. Am. J. Respir. Cell Mol. Biol. 2010; 43: 617-627.

31. Mikaelian I, Malek M, Gadet R, Viallet J, Garcia A, GirardGagnepain A, Hesling C, Gillet G, Gonzalo P, Rimokh $\mathrm{R}$, Billaud $\mathrm{M}$. Genetic and pharmacologic inhibition of mTORC1 promotes EMT by a TGF- $\beta$-independent mechanism. Cancer Res. 2013; 73: 6621-6631.

32. Wallin JJ, Guan J, Edgar KA, Zhou W, Francis R, Torres AC, Haverty PM, Eastham-Anderson J, Arena S, Bardelli A, Griffin S, Goodall JE, Grimshaw KM, et al. Active PI3K pathway causes an invasive phenotype which can be reversed or promoted by blocking the pathway at divergent nodes. PloS One 2012; 7: e36402.

33. Molinolo AA, Hewitt SM, Amornphimoltham P, Keelawat S, Rangdaeng S, Meneses García A, Raimondi AR, Jufe R, Itoiz M, Gao Y, Saranath D, Kaleebi GS, Yoo GH, et al. Dissecting the Akt/mammalian target of rapamycin signaling network: emerging results from the head and neck cancer tissue array initiative. Clin Cancer Res. 2007; 13: 4964-4973.

34. Molinolo AA, Marsh C, El Dinali M, Gangane N, Jennison K, Hewitt S, Patel V, Seiwert TY, Gutkind JS. mTOR as a molecular target in HPV-associated oral and cervical squamous carcinomas. Clin Cancer Res. 2012; 18: 2558-2568.

35. Wang Z, Martin D, Molinolo AA, Patel V, IglesiasBartolome R, Degese MS, Vitale-Cross L, Chen Q, Gutkind JS. mTOR co-targeting in cetuximab resistance in head and neck cancers harboring PIK3CA and RAS mutations. J Natl Cancer Inst. 2014; 106. 
36. Li L, Liu D, Qiu ZX, Zhao S, Zhang L, Li WM. The prognostic role of mTOR and p-mTOR for survival in non-small cell lung cancer: a systematic review and metaanalysis. PLoS One. 2015; 10: e0116771.

37. Ocana A, Vera-Badillo F, Al-Mubarak M, Templeton AJ, Corrales-Sanchez V, Diez-Gonzalez L, Cuenca-Lopez MD, Seruga B, Pandiella A, Amir E. Activation of the $\mathrm{PI} 3 \mathrm{~K} / \mathrm{mTOR} / \mathrm{AKT}$ pathway and survival in solid tumors: systematic review and meta-analysis. PLoS One. 2014; 9: e95219.

38. Beca F, Andre R, Martins DS, Bilhim T, Martins D, Schmitt F. p-mTOR expression is associated with better prognosis in luminal breast carcinoma. J Clin Pathol. 2014; 67: 961-967.

39. Rodrigo JP, García-Carracedo D, García LA, Menéndez S, Allonca E, González MV, Fresno MF, Suárez C, GarcíaPedrero JM. Distinctive clinicopathological associations of amplification of the cortactin gene at 11q13 in head and neck squamous cell carcinomas. J Pathol. 2009; 217: 516-523.

40. Rodrigo JP, Menéndez ST, Hermida-Prado F, ÁlvarezTeijeiro S, Villaronga MÁ, Alonso-Durán L, Vallina A, Martínez-Camblor P, Astudillo A, Suárez C, María García-Pedrero J. Clinical significance of Anoctamin-1 gene at 11q13 in the development and progression of head and neck squamous cell carcinomas. Sci Rep. 2015; 5: 15698.

41. Vivanco I, Sawyers CL. The phosphatidylinositol 3-Kinase AKT pathway in human cancer. Nature Rev. Cancer 2002; 2: 489-501.

42. Yuan TL, Cantley LC. PI3K pathway alterations in cancer: variations on a theme. Oncogene 2008; 27: 5497-5510.
43. Liu P, Cheng H, Roberts TM, Zhao JJ. Targeting the phosphoinositide 3-kinase pathway in cancer. Nature Rev. Drug Discov. 2009; 8: 627-644.

44. Simpson DR, Mell LK, Cohen EE. Targeting the PI3K/ AKT/mTOR pathway in squamous cell carcinoma of the head and neck. Oral Oncol. 2015; 51: 291-298.

45. Rodrigo JP, Heideman DA, García-Pedrero JM, Fresno MF, Brakenhoff RH, Díaz Molina JP, Snijders PJ, Hermsen MA. Time trends in the prevalence of HPV in oropharyngeal squamous cell carcinomas in northern Spain (1990-2009). Int J Cancer. 2014; 134: 487-92.

46. Rodrigo JP, Hermsen MA, Fresno MF, Brakenhoff RH, García-Velasco F, Snijders PJ, Heideman DA, GarcíaPedrero JM. Prevalence of human papillomavirus in laryngeal and hypopharyngeal squamous cell carcinomas in northern Spain. Cancer Epidemiol. 2015; 39: 37-41.

47. Menéndez ST, Rodrigo JP, Alvarez-Teijeiro S, Villaronga MÁ, Allonca E, Vallina A, Astudillo A, Barros F, Suárez C, García-Pedrero JM. Role of HERG1 potassium channel in both malignant transformation and disease progression in head and neck carcinomas. Mod. Pathol. 2012; 25: 1069-1078.

48. Garcia-Carracedo D1, Turk AT, Fine SA, Akhavan N, Tweel BC, Parsons R, Chabot JA, Allendorf JD, Genkinger JM, Remotti HE, Su GH. Loss of PTEN Expression is associated with poor prognosis in patients with intraductal papillary mucinous neoplasms of the pancreas. Clin. Cancer Res. 2013; 19: 6830-6841.

49. Rodrigo JP, Martínez P, Allonca E, Alonso-Durán L, Suárez C, Astudillo A, García-Pedrero JM. Immunohistochemical markers of distant metastasis in laryngeal and hypopharyngeal squamous cell carcinomas. Clin Exp Metastasis. 2014; 31: 317-325. 